\title{
Strategic Impact of a New Academic Endocrine Surgery Program
}

\author{
James E. Wiseman, MD ${ }^{1}$, Philip H. G. Ituarte, PhD $^{1}$, Roy S. Hwang, $\mathrm{MS}^{2}$, Ilan Safir, MS ${ }^{1}$, Jesse D. Pasternak, MD $^{3}$, \\ Karen Van Nuys, PhD ${ }^{1}$, and Michael W. Yeh, $\mathrm{MD}^{1}$ \\ ${ }^{1}$ Endocrine Surgical Unit, Department of Surgery, David Geffen School of Medicine at UCLA, Los Angeles, CA; \\ ${ }^{2}$ Cleveland Clinic Lerner College of Medicine, Cleveland Clinic Foundation, Cleveland, OH; ${ }^{3}$ Department of Surgery, \\ McMaster University, Hamilton, Ontario, Canada
}

\begin{abstract}
Background. A minority of medical centers possess a dedicated endocrine surgery program. Here we assess the short-term impact of a new endocrine surgery program on institutional case volumes and financial endpoints.

Methods. We studied all endocrine procedures performed over a 5-year period spanning the inception of the endocrine surgery program at UCLA. Institutional and statelevel data on patient geographic origin, discharges for endocrine diagnosis-related groups (DRGs), and hospitalside charges and costs were examined.

Results. Total endocrine case volume increased 112\% (264 to 559 cases annually) over the study period. The relative increase was greater for parathyroid (56 to 196, 250\%, $P<$ 0.0001 ) and adrenal ( 11 to $31,181 \%, P=0.06$ ) procedures compared to thyroid procedures (317 to $442,39 \%$ ). The endocrine case volume of nonspecialist surgeons remained stable over the study period. Growth in referrals arose from previously unrepresented zip codes and was associated with an increase in the mean distance traveled for care (2006, 44 miles vs. 2009, 92 miles, $P<0.01$ ). In each DRG, UCLA attained the top market position within one year of the program's inception, corresponding to an overall $27 \%$ increase in regional market share. Total hospital charges for endocrine DRGs rose $161 \%$ to $\$ 14.7$ million annually, while the cost of parathyroid surgery fell $34 \%(P<0.001)$.
\end{abstract}

Selected for Poster presentation competition at the 2010 annual meeting of the American Association of Endocrine Surgeons.

(C) The Author(s) 2011. This article is published with open access at Springerlink.com

First Received: 20 November 2010;

Published Online: 23 February 2011

J. E. Wiseman, MD

e-mail: jwiseman@mednet.ucla.edu
Conclusions. The establishment of an academic endocrine surgery program can cause fundamental shifts in referral patterns within a competitive, densely populated metropolitan environment. Hospitals should consider the inclusion of an endocrine surgery program in strategic planning initiatives.

Endocrine surgery is a maturing specialty that encompasses practitioners from both general surgery and otolaryngology backgrounds. ${ }^{1-3}$ Ample literature exists to support the relationship between surgeon volume and outcomes in complex and/or uncommon procedures. ${ }^{4-12}$ These data may underlie a national trend towards regionalization of care for complex surgery. ${ }^{13}$ While a growing number of surgeons identify endocrine diseases as an area of personal interest, a minority of medical centers possess dedicated, multidisciplinary endocrine surgery programs.

UCLA's multidisciplinary endocrine surgery program was established in 2006 for the purpose of concentrating experience regarding diseases of the thyroid, parathyroid, and adrenal glands on a select cadre of physicians with specific training/background in endocrine topics. We will use the term Endocrine Surgical Unit (ESU) to refer to subspecialty-trained surgeons within the larger program. Since its inception, we have prospectively measured the program's impact on clinical, educational, and hospital financial outcomes. In addition to improving coordination of care for complex diseases requiring multidisciplinary treatment, the ESU has developed a series of clinical protocols that objectively improve quality while reducing costs. ${ }^{14}$ Such systems of care are likely responsible for the superior outcomes generated by high-volume centers. ${ }^{15}$ Herein we describe the institutional effect the endocrine surgery program has had on surgical volume, referral patterns, market share, and hospital-side costs and charges. 


\section{SUBJECTS AND METHODS}

Data were obtained from medical cost and billing databases available from the UCLA Medical Center Financial Services department under IRB approval. Patient medical record numbers were masked to protect patient privacy in accordance with HIPAA standards. Electronic records from over 50,000 surgical patients admitted to the UCLA Westwood hospital between July 2002 and June 2009 (an 84-month period) were queried for endocrine-specific procedures. Using appropriate ICD-9-CM procedure codes, patients who received a unilateral thyroid lobectomy (ICD9-CM codes: 6.2, 6.31, 6.39), total thyroidectomy (ICD-9CM codes: 6.4, 6.51, 6.57), parathyroidectomy (ICD-9-CM codes: 6.81, 6.89), or adrenalectomy (ICD-9-CM codes: $7.21,7.22,7.29,7.3)$ were selected from the master file. Cases were excluded if patient records included a diagnosis of kidney transplantation, renal failure, or dialysis. Head and neck operations involving non-endocrine cancers were excluded, as were emergency operations. A subset of more common procedures, including appendectomy, laparoscopic cholecystectomy, colectomy, and hysterectomy, were also identified using appropriate ICD-9 codes. Cost, charges, and number of admissions for these procedures were aggregated to create an index of global institutional trends in surgical volumes and financial endpoints.

Demographic data collected included age, gender, race, ethnicity, health insurance, and five-digit zip code for primary residence. Medical information collected included diagnoses, procedures, treatment dates, total costs and charges. Distance traveled to UCLA was estimated by entering residential zip codes into a mileage calculator (Zip Code Distance Wizard, version 1.91). Data on market share was obtained from the California Office of Statewide Health Planning and Development. We analyzed institutional volume and financial trends by creating a cost index for common operations performed contemporaneously and involving similar nursing and ancillary staff. Control operations included laparoscopic cholecystectomy, open appendectomy, hemi-colectomy, and total hysterectomy. The following nonparametric tests were applied for data analysis: Wilcoxon rank-sum test, Kruskal-Wallis test, and contingency table tests. Data were analyzed by Stata/SE 9.2 (StataCorp, College Station, TX).

The study was conducted with the approval of the UCLA Institutional Review Board, protocol G08-11-01602.

\section{RESULTS}

From July 2002 to June 2009, a total of 46,807 unique admissions for surgical procedures occurred at UCLA Medical Center. Of these, 2,700 individuals were admitted for elective endocrine surgery, including 1,770 patients who underwent operations of the thyroid, 741 patients who underwent operations of the parathyroid glands, and 189 patients who underwent operations of the adrenal glands. These patients comprised the final study group. Table 1 displays the number of procedures by target organ per fiscal year from 2005-2009. Total endocrine case volume at our institution increased $112 \%$ (264 to 559 cases annually) over the study period. The relative magnitude of the volume increase was greater for parathyroid (56 to 196, $250 \%, P<0.001)$ and adrenal (11 to $31,181 \%, P=0.06$ ) procedures compared to thyroid procedures (197 to 334, $39 \%, P<0.001)$. In 2006, the first year of its existence, the ESU performed 35 of 285 (12.3\%) of all endocrine procedures at our institution. This proportion grew to 351 of $559(62.8 \%)$ in 2009. During this period of rapid growth within the ESU, the endocrine case volume of non-specialist surgeons within the institution remained stable, moving in parallel with overall surgical volumes (Fig. 1). No changes in UCLA network affiliations occurred during this time period.

An examination of the residential zip codes for those patients receiving endocrine surgical care at our institution demonstrated a significant increase in mean distance travelled per patient following the establishment of the ESU (2006, 44 miles vs. 2009, 92 miles, all cases, $P<0.0001$, Fig. 2). The increase in travel distance was most prominent for patients undergoing parathyroid surgery $(2006,41$ miles vs. 2009,149 miles, $P<0.001)$ but was also significant for thyroid surgery (2006, 42 miles vs. 2009, 60

TABLE 1 Comparison of case loads of ESU versus non-ESU surgeons, fiscal years 2005-2009

\begin{tabular}{|c|c|c|c|c|c|}
\hline & 2005 & 2006 & 2007 & 2008 & 2009 \\
\hline \multicolumn{6}{|l|}{ Total, $n(\%)$} \\
\hline Non-ESU & 264 & (100) & $250(87.7)$ & $228(56.2)$ & $187(41.4)$ \\
\hline \multicolumn{6}{|l|}{$208(37.2)$} \\
\hline ESU & - & $35(12.3)$ & $178(43.8)$ & $265(58.6)$ & $351(62.8)$ \\
\hline \multicolumn{6}{|c|}{ Thyroid, $n(\%)$} \\
\hline Non-ESU & 197 & (100) & $196(91.2)$ & $178(67.9)$ & $150(55.1)$ \\
\hline \multicolumn{6}{|l|}{$166(50.0)$} \\
\hline ESU & - & $19(8.8)$ & $84(32.1)$ & $122(44.9)$ & $166(50.0)$ \\
\hline \multicolumn{6}{|c|}{ Parathyroid, $n(\%)$} \\
\hline Non-ESU & 56 & (100) & $38(73.1)$ & $36(31.3)$ & $28(19.9)$ \\
\hline \multicolumn{6}{|l|}{$31(15.8)$} \\
\hline ESU & - & $14(26.9)$ & $79(28.7)$ & $113(80.1)$ & $165(84.2)$ \\
\hline \multicolumn{6}{|c|}{ Adrenal, $n(\%)$} \\
\hline Non-ESU & 11 & $(100)$ & $16(88.9)$ & $14(48.3)$ & $9(23.1)$ \\
\hline \multicolumn{6}{|l|}{$11(35.5)$} \\
\hline ESU & - & $2(11.1)$ & $15(51.7)$ & $30(76.9)$ & $20(64.5)$ \\
\hline
\end{tabular}

ESU Endocrine surgical unit 


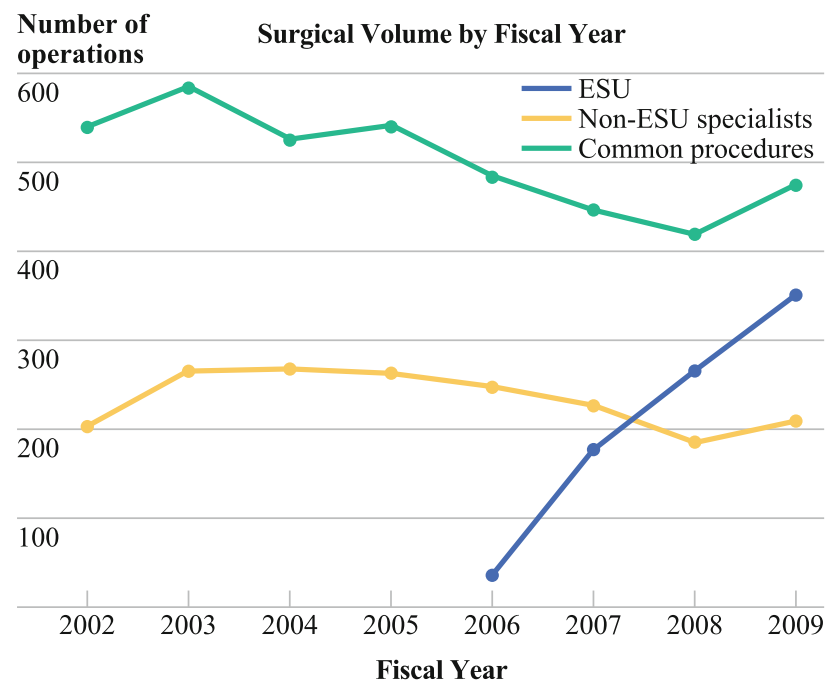

FIG. 1 Trends in absolute case volume of the ESU (blue line), as compared to non-specialist surgeons (yellow line), and select common operations (laparoscopic appendectomy, laparoscopic cholecystectomy, colectomy, hysterectomy; green line); fiscal years 2002-2009

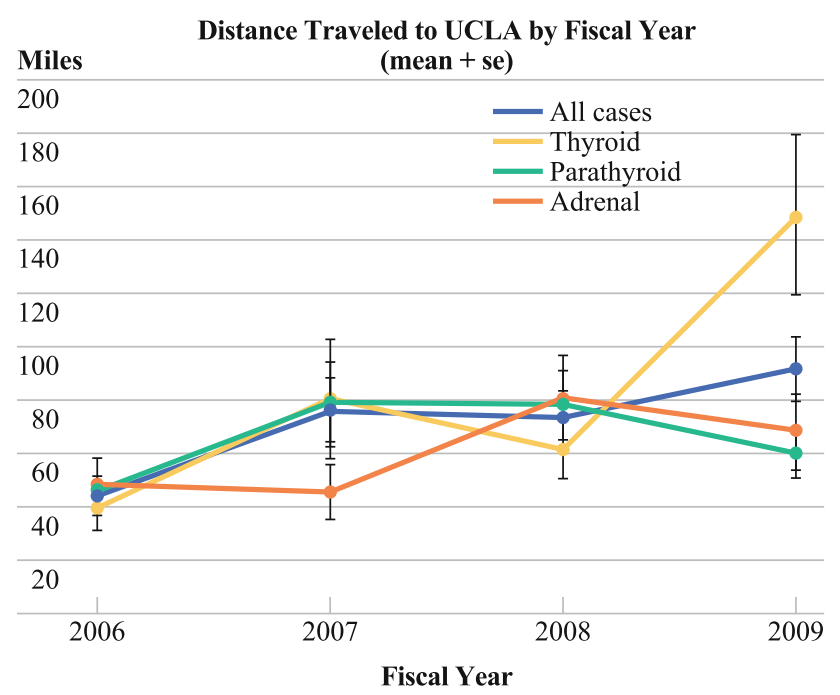

FIG. 2 Mean distance traveled for treatment by target organ, fiscal years 2006-2009

miles, $P<0.05$ ). Figure 3 features choropleth maps showing the distributions of patient origin by zip code from an eight-county region in Southern California (population 20 million, encompassing the following counties: Los Angeles, Orange, Riverside, San Bernardino, Ventura, Santa Barbara, Kern, San Luis Obispo) in 2005 and 2009. The total number of zip codes from which at least one patient was treated by the ESU in 2005 was 180. In 2009, 294 zip codes were represented $(P<0.001)$. Additionally, the number of zip codes yielding four or more patients increased from eight in 2005 to 35 in $2009(P<0.001)$.
Figure 4 shows the regional market shares of all institutions performing endocrine procedures. Over this time period, the annual number of endocrine procedures performed in the region grew $17 \%$, from 11,251 to 13,172 procedures. Beginning with 2005 , the year immediately prior to the establishment of the ESU, UCLA held the second-highest market share, performing 3.9\% (205 of 5247) of all endocrine operations in the region. By 2007, UCLA had achieved the top share at $6.4 \%$ (354 of 5522), and maintained this position through 2008 with a market share of $6.2 \%$ (341 of 5532). The slight decrease observed from 2007 to 2008 reflects the fact that approximately half of the ESU case volume was, at that time, shifted to the outpatient setting, which is not captured in the state-level market share data.

We identified 11 high-volume hospitals, performing 100 or more endocrine procedures annually, within the region. The near-doubling of UCLA's market share from 2003 to 2008 (3.2 to $6.2 \%)$ was associated with a rise in the total proportion of cases performed by high volume hospitals from 26.2 to $33.0 \%(P<0.0001)$.

The total costs incurred and charges billed for thyroid, parathyroid, and adrenal procedures were collected and adjusted to 2009 dollar values using the Consumer Price Index calculator. ${ }^{16}$ Annual charges for operations of the thyroid, parathyroid, and adrenal glands in aggregate exhibited a $161 \%$ increase from 2005 to 2009 , with total charges in 2009 reaching $\$ 14.7$ million. This occurred simultaneously with a steady decrease in the costs associated with endocrine procedures (13\% reduction compared to an index of common procedures), driven largely by a $34 \%$ decrease in the cost of parathyroid surgery per operation over the same time period $(P<0.001)$.

\section{DISCUSSION}

The precise number of multidisciplinary endocrine surgery programs in the nation is difficult to determine. Our informal survey of academic departmental websites identified 248 general surgery residency programs in the United States. Though we found that 134 of 248 departments (54.2\%) had one or more surgical faculty members expressing a specific interest in endocrine diseases, only 21 departments possessed dedicated endocrine centers.

Within the last decade, a growing body of literature has documented the association between volume and outcomes for complex surgical procedures. ${ }^{5,6,8,9,11,12}$ Several studies specifically examining endocrine procedures have demonstrated that high-volume surgeons achieve the lowest complication rates as well as shorter lengths of hospitalization. ${ }^{4,7,13}$ Despite these consistent findings, the great majority of endocrine procedures performed in the US 

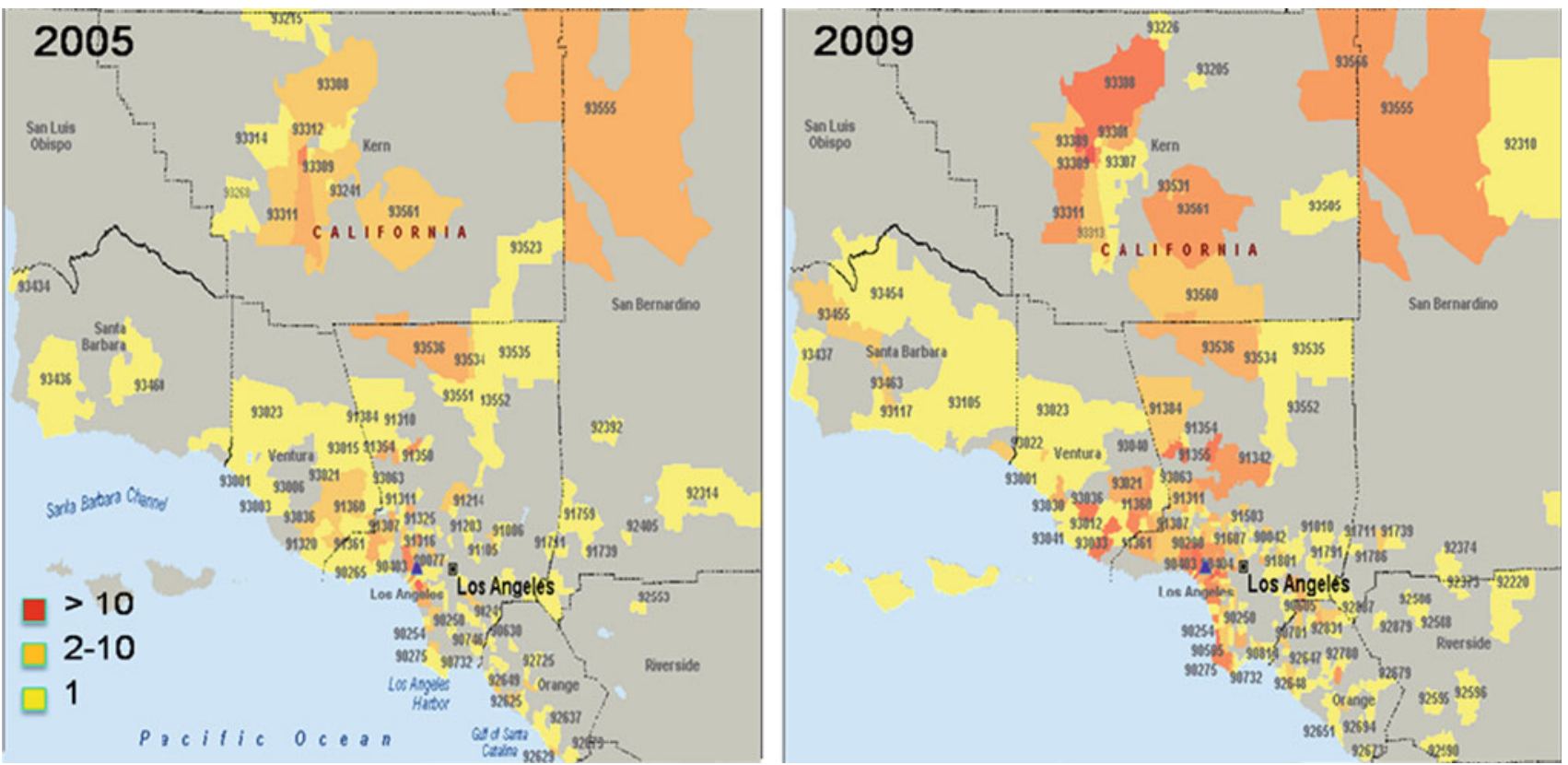

FIG. 3 Choropleth maps demonstrating the evolution of the distribution of zip code of origin for patients referred for surgical treatment of endocrine disorders at UCLA, fiscal years 2005-2009

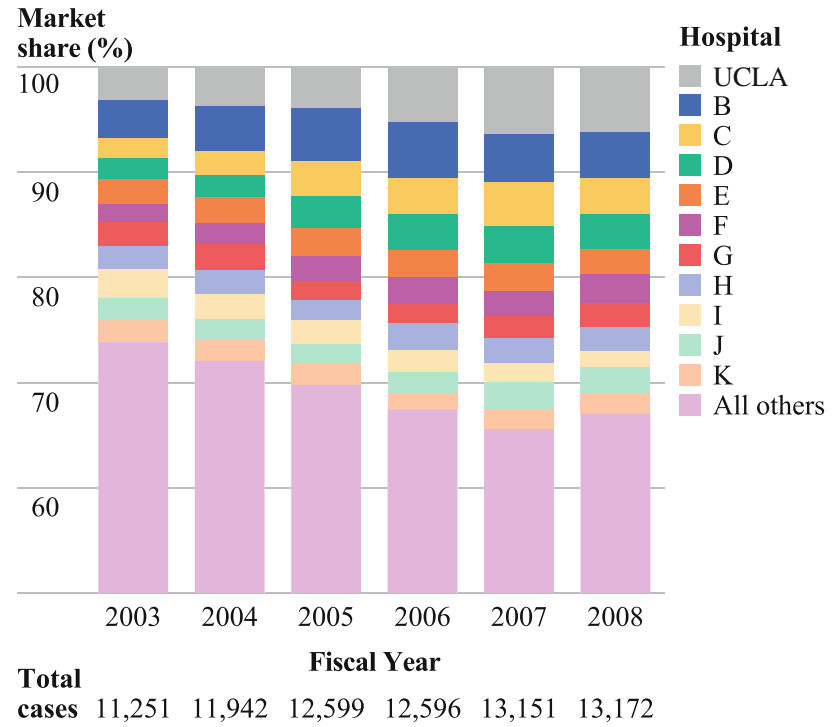

FIG. 4 Distribution of market share for endocrine operations, 8-county region (Los Angeles, Orange, Riverside, San Bernardino, Ventura, Santa Barbara, Kern), fiscal years 2002-2008

continue to be performed by low-volume surgeons. ${ }^{17}$ Proposed explanations for this discordance include limited opportunities for subspecialty training in endocrine surgery as well as limited patient access to high-volume surgeons, given their sporadic geographic distribution. ${ }^{2}$

Herein we have demonstrated in a prospective fashion that a newly established ESU can alter regional patterns in the delivery of endocrine surgical care. Within a short time frame, the ESU at UCLA concentrated a large volume of cases from a progressively widening geographic radius. The volume gains at this single center correlated with a $26 \%$ increase in the proportion of endocrine procedures performed at high-volume centers within the region. Changes of this nature are precisely those that would be predicted to improve the quality of surgical care provided. Indeed, we have described contemporaneous institutional improvements in clinical outcomes (reduced complication rates) and patient-level economic outcomes (reduced length of stay and hospital resource utilization) related to the growth of the ESU. ${ }^{14,18}$

The fact that most endocrine procedures in the nation are performed by low-volume surgeons represents an opportunity for the establishment of additional specialty centers. The magnitude of the volume-outcomes effect is pronounced for certain types of endocrine procedures, such as parathyroidectomy and thyroid cancer surgery, where initial referral to a specialty center is likely to yield lower complication rates and a reduced need for remedial surgery. ${ }^{4,19,20}$ We perceive that specialized endocrine surgery centers are currently growing at a rate much slower than that necessary to meet projected workforce needs. ${ }^{2} \mathrm{We}$ propose two potential reasons for this: (1) a perception that competition from neighboring hospitals would prohibit the establishment of a high-volume center, and (2) concern on the part of existing physicians within an institution that their surgical volumes would be adversely affected by a new specialty center. Indeed, we were motivated to present our data in order to debunk both of these arguments. 
Greater Los Angeles is the second largest population center in the United States and, according to most measures, one of the top ten largest urban areas in the world. ${ }^{21}$ In 2008, 13,172 endocrine procedures were performed across 208 hospitals in the region studied. Were these cases distributed uniformly, each hospital would perform 63 procedures, well less than the threshold required to achieve optimal outcomes. ${ }^{4}$ In other words, competition between hospitals within the region would likely counteract quality improvement. Our data demonstrate that intense competition from neighboring hospitals does not necessarily impede case volume concentration within a new specialty center.

Furthermore, the growth in volume within the ESU did not cannibalize volume from other practitioners within our institution. Instead, the added ESU cases appear to have come from an expansion of the geographic territory effectively served by the hospital for endocrine surgical needs. This point is of particular importance because existing practitioners are likely in a position to enable or block recruitment of the subspecialty-trained physicians necessary to create a new multidisciplinary endocrine surgery program. The slight decline in overall case volumes observed during 2007-2008 has been ascribed to the global economic downturn of the period.

The establishment of the ESU has also exerted a notable effect on average per-patient costs length of stay for endocrine procedures. This effect was primarily driven by two factors: the transition in 2008 of more than $90 \%$ of parathyroid operations to an outpatient surgery center, and the implementation of algorithm-based clinical pathways. ${ }^{14}$ Both of these actions have liberated valuable inpatient beds and operating rooms to serve the needs of an increasingly complex inpatient population.

In conclusion, our data demonstrate that the establishment of a dedicated endocrine surgical program at an academic hospital can lead to fundamental shifts in institutional referral patterns, resulting in higher operative volume. This ultimately contributes to the regionalization of care for complex surgical problems and the redistribution of regional market share. As such, academic medical centers should consider the establishment of a specialized endocrine surgery program a priority in the realm of strategic planning.

ACKNOWLEDGMENT The authors are grateful to the Earl Gales Family Foundation for their support of this project and to Nora Watson, Kari Smith, Beth Ten Pas, and Deborah Chandler for their assistance with data acquisition.

\section{CONFLICT OF INTEREST None.}

Open Access This article is distributed under the terms of the Creative Commons Attribution Noncommercial License which permits any noncommercial use, distribution, and reproduction in any medium, provided the original author(s) and source are credited.

\section{REFERENCES}

1. Pasieka JL. Kindred spirits of the endocrines: the training of the future endocrine surgeons. J Surg Oncol. 2005;89:202-5.

2. Sosa JA, Wang TS, Yeo HL, et al. The maturation of a specialty: workforce projections for endocrine surgery. Surgery. 2007; 142:876-83.

3. Thompson GB. The maturation of a specialty: workforce projections for endocrine surgery [editorial]. Surgery 2007;142: 884-6.

4. Stavrakis AI, Ituarte PHG, Ko CY, Yeh MW. Surgeon volume as a predictor of outcomes in inpatient and outpatient endocrine surgery. Surgery 2007;142:887-99.

5. Birkmeyer JD, Siewers AE, Finlayson EVA, et al. Hospital volume and surgical mortality in the United States. $N$ Engl J Med. 2002;346:1128-37.

6. Martling A, Cedarmark B, Johansson H, Rugqvist L, Holm T. The surgeon as a prognostic factor after the introduction of total mesorectal excision in the treatment of rectal cancer. Br J Surg. 2002;89:1008-13.

7. Sosa JA, Bowman HM, Tielsch JM, Powe NR, Gordon TA, Udelsman R. The importance of surgeon experience for clinical and economic outcomes for thyroidectomy. Ann Surg. 1998; 228:320-30.

8. Birkmeyer JD, Stukel TA, Siewers AE, Goodney PP, Wennberg DE, Lucas FL. Surgeon volume and operative mortality in the United States. N Engl J Med. 2003;349:2117-27.

9. Wijns W, Kolh PH. Experience with revascularization procedures does matter: low volume means worse outcome. Eur Heart J. 2010;31:1954-7.

10. Hudorovic N, Vucetic B, Lovricevic I. The evidence for volumeoutcome relationships in thoracic aortic surgery. Eur J Cardiothorac Surg. 2010;37:248-9.

11. Begg CB, Cramer LD, Hoskins WJ, Brennan MF. Impact of hospital volume on operative mortality for major cancer surgery. JAMA. 1998;280:1747-51.

12. Finlayson EVA, Goodney PP, Birkmeyer JD. Hospital volume and operative mortality in cancer surgery: a national study. Arch Surg. 2003;138:721-5.

13. Boudourakis LD, Roman SA, Sosa JA, Wang TS, Desai R. Evolution of the surgeon-volume, patient outcome relationship. Ann Surg. 2009;251:992.

14. Wiseman J, Mossanen M, Ituarte P, Bath J, Yeh M. An algorithm informed by the parathyroid hormone level reduces hypocalcemic complications of thyroidectomy. World J Surg. 2010;34:532-7.

15. Khuri SF. Safety, quality, and the National Surgical Quality Improvement Program. Am Surg. 2006;72:994-8.

16. Consumer price index calculator. Available at: http://data.bls. gov/cgi-bin/cpicalc.pl. Accessed 29 March 2009.

17. Saunders BD, Wainess RM, Dimick JB, Doherty GM, Upchurch GR, Gauger PG. Who performs endocrine operations in the United States? Surgery. 2003;134:924-31.

18. Kulkarni RP, Ituarte PHG, Gunderson D, Yeh MW. Clinical pathways improve hospital resource use in endocrine surgery. $J$ Am Coll Surg. 2011;212:35-41.

19. Kouvaraki MA, Lee JE, Shapiro SE, Sherman SI, Evans DB. Preventable reoperations for persistent and recurrent papillary thyroid carcinoma. Surgery. 2004;136:1183-91.

20. Mitchell J, Milas M, Barbosa G, Sutton J, Berber E, Siperstein A. Avoidable reoperations for thyroid and parathyroid surgery: effect of hospital volume. Surgery 2008;144:899-907.

21. Forstall RL, Greene RP, Pick JB. Which are the largest? Why lists of major urban areas vary so greatly. Tijdschr Econ Soc Geogr. 2009;100:277-97. 